\title{
Congruence and Divisibility: Divisibility Criteria for Positive Integers
}

\author{
Sisay Tadesse \\ Sisay Tadesse, Department of Mathematics, College of Natural and Computational Sciences, Wolaita Sodo \\ University, PO box 138, Wolaita Sodo, St. 7, Ethiopia
}

\begin{abstract}
In this paper we deal with divisibility criteria for any integer in decimal system. In the development of these criteria we use facts from congruence theory: as modular Arithmetic, linear congruences, and some important properties of divisibility and congruence. Then, we give general divisibility criteria for the two classes of positive integers. The divisibility criteria for the first class of divisors is written down as a linear form in which the decades and the units digits of the test integer are involved in such a way that the co-efficient of the decades takes one and that of the units digit is an integer formed by a parameter, which is the solution of the linear congruence describing the co-primality of the divisor and the base of the underlying number system. This divisibility parameter is not unique, but each yields a unique criterion. Finally, we apply the rule giving a couple of examples and make a conclusion which summarizes the general divisibility test in terms of the two classes of divisors.

Keywords: co-prime, modular Arthmetics, linear congruences, divisibility criteria, fundamental theorem of arthmetics

DOI: $10.7176 / \mathrm{JNSR} / 9-7-06$

Publication date: April $30^{\text {th }} 2019$

\section{Introduction}

Divisibility rules are designed to answer the question of divisibility of an integer a by a divisor integer without actually performing division. There are lots works that had been done in the field of number theory. But in the area of divisibility very little attention has been given. Although, for checking that a given integer is a multiple of any other integer is still time taking, we have some algorithms, such as Euclid's algorithm, which is one of the preeminent methods ever known regarding the underlying concept. Till the date there was no a feasible generalized test for divisibility. Here are some facts of congruence theory, which is an important tool in number theory, besides handling related problems as solving congruence equations, remainder problems and the like, it is being used in the development of a generalized test of divisibility. The basic facts that are to be used in this paper are linear congruences and their properties along with modular Arthmetics and the Fundamental theorem of Arthmetics. In section four ,we show an application for the main result. A conclusion is given in the last section of this paper
\end{abstract}

\section{Congruence and its properties, and basic notions}

\subsection{Congruence}

Definition2.1. If $a$ and $b$ are integers; the notation $a \equiv(\bmod m)$ (" $a$ is congruent to $b \bmod m$ ") means that $a$ and $b$ share the same remainder with respect to integer division by $m$, or, equivalently, that $m \mid b-a$.

Definition 2.2. Let $a, b, m$ be integers with $m>0$, then we say $a$ is congruent to $b$ modulo $\mathrm{m}$ iff $m \mid a-b$. Symbolically, $a \equiv b(\bmod m)$.

Examples 2.1. Congruence between two integers

a) $\quad 3 \equiv 5 \bmod 2$,

b) $\quad 23 \equiv 37(\bmod 7)$.

Remark 2.1. Here, we see why the above two definitions are equivalent. If $a$ and $b$ have the same remainder (mod $m$ ), then $a=m q_{1}+r$ for some integer $q_{1}$ and some $0 \leq r<m$, and $b=m q_{2}+r$ for some integer $q_{2}$ and the same $r$. Therefore,

$$
b-a=m\left(q_{2}-q_{1}\right)
$$

, which means that $m \mid b-a$. Conversely, if $m \mid b-a$, then $b-a=m q$ for some integer $q$. Then let amod $m=r$.It follows that $a=m q_{1}+r$ for some integer $q_{1}$. But then,

$$
b=a+m q=m q_{1}+r+m q=m q+m q_{1}+r
$$

Since $r$ is the remainder of $(\bmod m), 0 \leq r<m$, and therefore, since $b=m q+q 1+r, r$ is also the remainder of $b(\bmod m)$.

The condition $m \mid b-a$ can also be expressed as $b=a+m q$ for some integer $q$.Therefore, $a$ is congruent to $b$ mod $m$ precisely if the difference of $a$ and $b$ is a multiple of $m$.An observation that will be useful later is that $a \equiv(a \bmod m)(\bmod m)$. This follows directly from the definition. 


\subsection{Properties of Congruence}

We now study how congruence interacts with the arithmetic operations of addition and multiplication.

Theorem 2.1. if $a \equiv b(\bmod m)$ and $c \equiv d(\bmod m)$, then

i. $\quad a+c \equiv b+d(\operatorname{modm})$

ii. $\quad a c \equiv b d(\operatorname{modm})$

Proof: As we just stated on the previous remark, the assumptions of the theorem are equivalent to $b=a+m q_{1}$ for some integer $q_{1}$ and $d=c+m q_{2}$ for some integer $q_{2}$. By Then it follows that $+d=a+c+m q_{1}+m q_{2}=a+$ $c+m\left(q_{1}+q_{2}\right)$. That proves the first conclusion of the theorem. Similarly, we get $b d=a c+m q_{1} c+q_{2} a+$ $q_{1} q_{2}$. That proves the second conclusion of the theorem.

It is a consequence of this theorem that in any computation of a remainder of some additive and/or multiplicative combination of integers, the integers involved can be reduced to remainders first. We will explain this later.

Definition 2.3. We define relation ' $\equiv^{\prime}$ as $a \equiv b(\bmod m)$ iff $m \mid a-b$. Such a relation is called a congruence relation.

Theorem 2.2. The congruence relation satisfies the following properties: for $a, b, c \in Z$ (i.e. an equivalence relation)

a. $\quad a \equiv a(\bmod m)($ Reflexive $)$

b. $\quad a \equiv b(\bmod m)$ implies $b \equiv a(\bmod m)($ symmetric)

c. $\quad a \equiv b(\bmod m)$ and $b \equiv c(\bmod m)$ then $a \equiv c(\bmod m)($ transitive $)$

Proof: left to the reader.

Theorem 2.3. If $a_{1} \equiv b_{1}(\bmod n)$ and $a_{2} \equiv b_{2}(\bmod n)$, or if $a \equiv b(\bmod n)$, then:

a. $\quad a+k \equiv b+k(\bmod n)$ for any integer $k$ (compatibility with translation)

b. $\quad k a \equiv k b(\bmod n)$ for any integer $k$ (compatibility with scaling)

c. $\quad a_{1}+a_{2} \equiv b_{1}+b_{2}(\bmod n)$ (compatibility with addition)

d. $\quad a_{1}-a_{2} \equiv b_{1}-b_{2}(\bmod n)$ (compatibility with subtraction)

e. $\quad a k \equiv b k(\bmod n)$ for any non-negative integer $k$ (compatibility with exponentiation)

f. $\quad p(a) \equiv p(b)(\bmod n)$, for any polynomial $p(x)$ with integer coefficients (compatibility with polynomial evaluation)

Corollary2.1. For cancellation of common terms, we have the following rules:

a. If $a+k \equiv b+k(\bmod n)$ for any integer $k$, then $a \equiv b(\bmod n)$

$b$. If $k a \equiv k b(\bmod n)$ and $k$ is coprime with $n$, then $a \equiv b(\bmod n)$

Definition 2.4. The modular multiplicative inverse is defined by the following rules:

There exists an integer denoted $a^{-1}$ such that $a a^{-1} \equiv 1(\bmod n)$ if and only if $a$ is coprime with $n$. This Integer $a^{-1}$ is called a modular multiplicative inverse of $a$ modulo $n$.

$\circ \quad$ If $a \equiv b(\bmod n)$ and $a^{-1}$ exists, then $a^{-1} \equiv b^{-1}(\bmod n)$ (compatibility with multiplicative inverse, and, if $a=b$, uniqueness modulo $n$ ).

In particular, if $p$ is a prime number then $a$ is coprime with $p$ for every $a$ such that $0<a<p$. Thus, a multiplicative inverse exists for all $a$ that are not congruent to zero modulo $p$.

\subsection{Linear congruence}

Definition 2.5. The congruence of the form $a x \equiv b(\bmod m)$ is called a linear congruence with one variable $\mathrm{x}$.

Example 2.2. Consider $2 x-5 \equiv 1(\bmod 3)$. It is an example of a linear congruence which can be reduced to $2 x \equiv 6(\bmod 3)$.

Definition 2.6. By a solution of the linear congruence $a x \equiv b(\bmod m)$, we mean $x_{0}$ such that $a x_{0} \equiv b(\bmod m)$. Example 2.3. The solution of the linear congruence in the above example is 3 .

Theorem 2.4. The linear congruence $a x \equiv b(\bmod m)$ has solution if and only if $\operatorname{gcd}(a, m) \mid b$.

Remark 2.2. If $a x \equiv b(\bmod n)$ and a is coprime to $n$, the solution to this linear congruence is given by $x \equiv a^{-1}$ $b(\bmod n)$.

Example 2.4. Solve a linear congruence: find a solution to $8 \mathrm{x} \equiv 1(\bmod 11)$. If there is an answer, it can be represented by one of $0,1,2, \ldots, 10$, so we can just run through the possibilities:

\begin{tabular}{|c|c|c|c|c|c|c|c|c|c|c|c|}
\hline$x \bmod 11$ & 0 & 1 & 2 & 3 & 4 & 5 & 6 & 7 & 8 & 9 & 10 \\
\hline $8 \times \bmod 11$ & 0 & 8 & 5 & 2 & 10 & 7 & 4 & 1 & 9 & 6 & 3 \\
\hline
\end{tabular}

The only solution is $7 \bmod 11: 8 \times 7=56 \equiv 1 \bmod 11$. This means 7 and 8 are multiplicative inverses in $Z_{11}$. This problem concerns finding an inverse for 8 modulo 11 . We can find multiplicative inverses for every nonzero element of $Z_{11}$ :

\begin{tabular}{|l|l|l|l|l|l|l|l|l|l|l|}
\hline $\mathrm{x}$ & 1 & 2 & 3 & 4 & 5 & 6 & 7 & 8 & 9 & 10 \\
\hline$x^{-1}$ & 1 & 6 & 4 & 3 & 9 & 2 & 8 & 7 & 5 & 10 \\
\hline
\end{tabular}

Check in each case that the product of the numbers in each column is 1 in $Z_{11}$. 
Example 2.5.

Find a solution to $8 x \equiv 1(\bmod 10)$. We run through the standard representatives for $\left.Z_{10}\right)$, and find no answer:

\begin{tabular}{|l|l|l|l|l|l|l|l|l|l|l|l|}
\hline $\mathrm{x} \bmod 10$ & 0 & 1 & 2 & 3 & 4 & 5 & 6 & 7 & 8 & 9 & 10 \\
\hline $8 \mathrm{x} \bmod 10$ & 0 & 8 & 6 & 4 & 2 & 0 & 8 & 6 & 4 & 2 & 0 \\
\hline
\end{tabular}

In retrospect, we can see a priori why there shouldn't be an answer. If $8 x \equiv 1 \bmod 10$. for some integer $\mathrm{x}$, then we can lift the congruence up to $Z$ in the form $8 x+10 y=1$ for some $y \in Z$. But this is absurd: $8 x$ and $10 y$ are even, so the left side is a multiple of 2 but the right side is not.

Example 2.6. The linear congruence $6 x+1 \equiv 4(\bmod 15)$ has three solutions! In the following table we can see the solutions are 3,8 , and 13:

\begin{tabular}{|r|r|r|r|r|r|r|r|r|r|r|r|r|r|r|r|}
\hline $\mathrm{x} \bmod 15$ & 0 & 1 & 2 & 3 & 4 & 5 & 6 & 7 & 8 & 9 & 10 & 11 & 12 & 13 & 14 \\
\hline $6 \mathrm{x}+1 \bmod 15$ & 1 & 7 & 13 & 4 & 10 & 1 & 7 & 13 & 4 & 10 & 1 & 7 & 13 & 4 & 10 \\
\hline
\end{tabular}

These examples show us that a linear congruence $a x \equiv b \bmod m$ doesn't have to behave like real linear equations: there may be no solutions or more than one solution. In particular, taking $b=1$, we can't always find a multiplicative inverse for each nonzero element of $Z_{m}$. The obstruction to inverting 8 in $Z_{10}$ can be extended to other cases in the following way.

Theorem 2.4. For integers a and $m$, the following are equivalent:

i. There is a solution $\mathrm{x}$ in $\mathrm{Z}$ to $a x \equiv b \bmod m$,

ii. $\quad$ There are solutions $\mathrm{x}$ and $\mathrm{y}$ in $\mathrm{Z}$ to $\mathrm{ax}+\mathrm{my}=1, a$ and $m$ are relatively prime.

Proof. Suppose $a x \equiv b \bmod m$ for some $x \in Z$. Then, $m y=(1-a x)$, so there is some $y \in Z$ such that $m y=1-a x$, so $\mathrm{ax}+\mathrm{my}=1$ :

\subsection{Modular Arithmetic}

In mathematics, modular arithmetic is a system of arithmetic for integers, where numbers "wrap around" upon reaching a certain value called the modulus. The modern approach to modular arithmetic was developed by Carl Frederich Gauss in his book Disquisitiones Arithmeticae in 1801.

In some applications, we are only interested in the remainder of some arithmetic operation. A familiar use of modular arithmetic is in the 12 hours clock, in which the day is divided in two 12 hours periods. For instance, if the time is 10:00 now, then after 5 hours it will be 3:00. Usual addition would suggest that the later time should be $10+5=15$ but this is not the case because clock time "wrap around" every 12 hours. Because the hour number starts over after it reaches 12, this is arithmetic modulo 12. According to definition of congruence, 12 is congruent not only to itself, but also to 0 , so the time is called " $12: 00$ " could also be called " $0: 00$ ", since $12 \equiv 0(\bmod 12)$.

Performing addition and multiplication only on the set of integers from 0 to $m-1$, called $\mathbb{Z}_{m}$ and reducing each sum or product $\bmod m$ is called modular arithmetic (in $\mathbb{Z}_{m}$ ). For example, in $\mathbb{Z}_{m}, 4$ plus 3 is 2 , and 2 times 3 is 1 .

Lemma 2.1. Let $a, b, m \in \mathbb{N}$, then if $a+b \equiv 0(\bmod m)$, then $a \equiv 0(\bmod m)$ iff $b \equiv 0(\bmod m)$.

As it is easy to verify, the proof is left to the reader.

We took what appears to be a detour through equivalence relations because those three properties allow us to define addition, subtraction, and multiplication for congruences. Addition, subtraction, and multiplication work exactly the same way as they do with integers with the only constraint being that addition, subtraction, and multiplication is only allowed when the congruences have the same moduli.

Proposition 2.1. Suppose we have some and then $a_{1} \equiv b_{1}(\bmod c)$ and $a_{2} \equiv b_{2}(\bmod c)$ then

i. $\quad a_{1} \pm a_{2} \equiv b_{1} \pm b_{2}(\bmod c)$ (Addition and Subtraction)

ii. $\quad a_{1} \cdot a_{2} \equiv b_{1} \cdot b_{2}(\bmod c)$ (Multiplication)

Example 2.7. Since $23 \equiv 3(\bmod 4)$ and $6 \equiv 2(\bmod 4)$ the following are true:

1. $(23+6) \equiv(2+3)(\bmod 4)=29 \equiv 5(\bmod 4)=29 \equiv 1(\bmod 4)($ Addition $)$

2. $(23-6) \equiv(2-3)(\bmod 4)=17 \equiv-1(\bmod 4)=17 \equiv 3(\bmod 4)$ (Subtraction)

$3 .(23 * 6) \equiv(2 * 3)(\bmod 4)=138 \equiv 6(\bmod 4)=138 \equiv 2(\bmod 4)($ Multiplication $)$

Remark 2.3. Just as we cannot divide by zero in normal arithmetic, division for modular congruences is only permissible under certain Circumstances.

Proposition 2.2. If $b d_{1} \equiv b d_{2}(\bmod c)$ and if ${ }^{1} \operatorname{gcd}(b, c)=1$ then $d_{1} \equiv d_{2}(\bmod c)$.

Example 2.8. Consider $14 \equiv 4(\bmod 10)$.Here, we cannot divide both sides by two because $7 \not \equiv 2(\bmod 10)$. In other words, $14 \equiv 4(\bmod 10)$ fails to divide by 2 because both 2 and 10 are divisible by 2 . Again, we can only divide provided that there are no common divisors between the number we are trying to divide by and the modulus. Note that if the modulus is a prime number then division is defined for all divisors.

\footnotetext{
${ }^{1}$ The gcd $(b, c)$ means the greatest common divisor for - the greatest number that divides both and. When the greatest common divisor of two numbers is 1 that means there are no other such divisors.
} 


\subsection{Fundamental theorem of Arithmetic}

Every natural number is built, in a unique way, out of prime numbers. Note that primes are the products with only one factor and 1 is the empty product.

Theorem 2.5. Every natural number can be written as a product of primes uniquely up to order.

Proof: An interested reader can establish the proof of this theorem using Mathematical induction and for the uniqueness part, also using proof by contradiction.

Example 2.9. Write the natural number $n=2775$ as a product of distinct primes. The prime factorization of $2775=$ $3 \times 5^{2} \times 37$.

\section{Main Result}

As far as our concern that we are developing a test for divisibility of integers co-prime to 10

Proposition 3.1. Let $m$ be a positive integer co-prime to 10 , then there is an integer $\mathrm{x}$ such that $10 x \equiv 1(\bmod m)$.

Proof: $\operatorname{As} \operatorname{gcd}(m, 10)=1$, then by $\mathrm{GCD}^{1}$-Theorem there are integers $\mathrm{x}$ and $\mathrm{y}$ such that $10 x+m y=1$. But in view of congruence theory, we obtain that $10 x \equiv 1(\bmod m)$ Thus, our main task here is finding such an integer $\mathrm{x}$ satisfying the congruence equation in the above proposition. In performing this task of developing the criteria we require to solve the linear congruence using cancellation law in congruences. Clearly, it has solution because it satisfies the existence theorem for solution of linear congruences. Theorem 3.1. Let $x_{0}$ be a solution of the congruence (1), then the solution set of (1) is given by $\left\{x_{0}+m k: k \in\right.$ $Z\}$.

Proof: Suppose $x_{0}$ is a solution of the linear congruence, $10 x \equiv 1(\bmod m)$ then any solution $x$ of the congruence is given by $x \equiv x_{0}(\bmod m)$. Thus, the solution set of the congruence is $\left\{x_{0}+m k: k \in Z\right\}$.

Remark 3.1.

i. There are infinitely many integer solutions which are multiplicative inverses to 10 .

ii. As $m$ is co-prime to 10 , the possible unit digit of $m$ takes one of the values $1,3,7$ and 9 .

Theorem 3.2. Let $\mathrm{A}=\sum_{i=0}^{n} 10^{i} a_{i}$ be a test number and $\mathrm{m}$ a positive integer with $\operatorname{gcd}(m, 10)=1$, then $A \equiv$ $0(m d m)$ iff $B \equiv 0(\bmod m)$, where $\mathrm{B} \ll \mathrm{A}$ and $\mathrm{B}=\sum_{i=1}^{n} 10^{i-1} a_{i}+\left(\frac{1+m k}{10}\right) \cdot a_{0}, k \in Z^{-}$.

Proof: suppose $\mathrm{A}=\sum_{i=0}^{n} 10^{i} a_{i}$ a number in decimal number system and $\mathrm{m}$ is a positive integer with $\operatorname{gcd}(\mathrm{m}, 10)=$ 1(i.e. $\mathrm{m}$ is co-prime to 10). Let $\mathrm{b}$ and $a_{0}$ be the number of decades and units respectively, so that $A=10 b+a_{0}$. Now suppose $\mathrm{A}$ is divisible by $\mathrm{m}$ (i.e. in view of congruence $\mathrm{A} \equiv 0(\bmod \mathrm{m})$. Then, we have, $10 b \equiv$ $-a_{0}(\bmod m)$. As $\operatorname{gcd}(10, m)=1$, by proposition 3.1 . There is an integer $\mathrm{x}$ such that $10 x \equiv 1(\bmod m)$.Then in view of division algorithm, we have $10 x=1+m k$, for some integer $\mathrm{k}$. Thus, $x=\frac{1+m k}{10}$ (where $\mathrm{x}$ is a modular multiplicative inverse of 10 in $\left.Z_{m}\right)$

Consider $10 b \equiv-a_{0}(\bmod m)$. Then, evidently, we obtain $10 x b \equiv-x a_{0}(\bmod m) \Rightarrow$

$b \equiv-x a_{0}(\bmod m) \Longrightarrow b+x a_{0} \equiv 0(\bmod m)$. Now, let $\mathrm{B}=b+x a_{0}$, then $B=\sum_{i=1}^{n} 10^{i-1} a_{i}+\left(\frac{1+m k}{10}\right) \cdot a_{0} \equiv$ $0(\bmod m)$.

Again, suppose $\sum_{i=1}^{n} 10^{i-1} a_{i}+\left(\frac{1+m k}{10}\right) \cdot a_{0} \equiv 0(\bmod m)$ and $\operatorname{gcd}(10, m)=1$, then we show that $\mathrm{A} \equiv$ $0(\bmod \mathrm{m})$. Consider $\sum_{i=1}^{n} 10^{i-1} a_{i}+\left(\frac{1+m k}{10}\right) \cdot a_{0} \equiv 0(\bmod m)$ then as $10 x \equiv 1(\bmod m)$, we obtain $10 x \cdot \sum_{i=1}^{n} 10^{i-1} a_{i}+x \cdot a_{0} \equiv 0(\bmod m)$

$\Rightarrow x\left(\sum_{i=1}^{n} 10^{i} a_{i}+a_{0}\right) \equiv 0(\bmod m)$. Then in view of cancellation law, as $\mathrm{x}$ is coprime to $\mathrm{m}$, we have $\sum_{i=1}^{n} 10^{i} a_{i}+a_{0} \equiv 0(\bmod m) \Rightarrow \sum_{i=0}^{n} 10^{i} a_{i} \equiv 0(\bmod m) \Rightarrow \mathrm{A} \equiv 0(\bmod m)$.

Remark 3.2. According to the fact (i) in Remark 3.1., the divisibility criterion for $m$ is not unique. For instance, for $\mathrm{m}=9$, besides what is given under special divisibility criteria for integers co-prime to 10 , we have at least one criterion $\mathrm{b}-17 a_{0} \equiv 0(\bmod 9)$.

\subsection{Special divisibility criteria for integers coprime to $\mathbf{1 0}$}

From the generalized divisibility criteria we extracted the special ones for few positive integers discussed as follows:

Let $\mathrm{A}$ be a test number and $\mathrm{m}$ be a composite positive integer co prime to 10 .

Suppose, $A=10^{n} a_{n}+10^{n-1} a_{n-1}+\cdots+10^{2} a_{2}+10^{1} a_{1}+a_{0}$. Let $\mathrm{b}=a_{n} a_{n-1} \ldots a_{2} a_{1}$ (i.e. decades) and $a_{0}$ is units digit. Then $A=10 b+a_{0}$.

Here we give Divisibility criteria for 9, 21, 27 and 33 as follows

${ }^{1}$ GCD-Theorem: For integers $a_{1}, a_{2}, \ldots, a_{n}$, there is a positive integer $d=\operatorname{gcd}\left(a_{1}, a_{2}, \ldots, a_{n}\right)$ and there are some integers $x_{1}, x_{2}, \ldots, x_{n}$ such that $d=\sum_{i=1}^{n} a_{i} x_{i}$. 


\section{Proposition 3.2. Divisibility criterion for 9}

Suppose $\mathbf{A} \equiv 0(\bmod 9) \Leftrightarrow \mathrm{B}=\sum_{i=1}^{n} 10^{i} a_{i}+\left(\frac{1+9 k}{10}\right) \cdot a_{0}, k=-9$ by the above theorem, we obtain that $B=$ $b-8 a_{0} \equiv 0(\bmod 9)$.

Proposition 3.3. Divisibility criterion for 21

Suppose $\mathrm{A} \equiv 0(\bmod 21) \Leftrightarrow \mathrm{B}=\sum_{i=1}^{n} 10^{i} a_{i}+\left(\frac{1+21 k}{10}\right) \cdot a_{0}, k=-1$, then by the above theorem, we obtain that $B=b-2 a_{0} \equiv 0(\bmod 21)$.

Proposition 3.4. Divisibility criterion for 27

Suppose $\mathbf{A} \equiv 0(\bmod 27) \Rightarrow 10 b+a_{0} \equiv 0(\bmod 27) \Leftrightarrow \mathrm{B}=\sum_{i=1}^{n} 10^{i} a_{i}+\left(\frac{1+27 k}{10}\right) \cdot a_{0}, k=-3$. Thus, we obtain that $b-8 a_{0} \equiv 0(\bmod 27)$.

Proposition 3.5. Divisibility criterion for 33

Suppose $\mathbf{A} \equiv 0(\bmod 33) \Rightarrow 10 b+a_{0} \equiv 0(\bmod 33) \Leftrightarrow \mathrm{B}=\sum_{i=1}^{n} 10^{i} a_{i}+\left(\frac{1+33 k}{10}\right) \cdot a_{0}, k=-7$, Thus, we obtain that $b-23 a_{0} \equiv 0(\bmod 33)$.

3.2. Special divisibility criteria for integers not coprime to 10

In this subsection we discuss divisibility criteria for those positive integers not relatively prime to 10. One may ask for what these integers are. Obviously, they are those integers which are multiples of 2 and /or 5 and their powers. So, here we need to use the fundamental theorem of arithmetic in expressing the underlying number (divisor) as a product of distinct primes.

Lemma 3.2.1. Let $A=\sum_{i=0}^{n} 10^{i} a_{i}, a_{n} \neq 0$ be given integer. Then $A \equiv 0\left(\bmod 2^{n}\right)$ iff $\left(a_{n-1} a_{n-2} \ldots a_{1} a_{0}\right)_{t e n} \equiv 0\left(\bmod 2^{n}\right)$.

Lemma 3.2.2. Let $A=\sum_{i=0}^{n} 10^{i} a_{i}, a_{n} \neq 0$ be given integer. Then $A \equiv 0\left(\bmod 5^{n}\right)$ iff $\left(a_{n-1} a_{n-2} \ldots a_{1} a_{0}\right)_{t e n} \equiv 0\left(\bmod 5^{n}\right)$.

Proposition 3.6. Let $m=2^{\alpha} .5^{\beta} . h$ with $\mathbf{h}$ co-prime to 10 (i.e. $h=p_{1}^{\theta_{1}} p_{2}^{\theta_{2}} \ldots p_{r}^{\theta_{r}}$ with each $p_{i} \neq 2,5$ ) and $\alpha, \beta$ are non-negative integers, and $\mathrm{A}$ be a test number then $A \equiv 0(\bmod m)$ iff $\equiv 0\left(\bmod 2^{\alpha}\right), A \equiv$ $0\left(\bmod 5^{\beta}\right)$ and $A \equiv 0(\bmod h)$.

\section{Applications}

In this section we shall utilize the divisibility criteria to show some examples.

Example 4.1.

Consider a number $A=6253$.we verify whether $A$ is divisible by $m=481$. Since the last digit of $m$ is 1 , the criterion is given by $\mathrm{B}=\sum_{i=1}^{n} 10^{i} a_{i}+\left(\frac{1+481(-1)}{10}\right) \cdot a_{0}, \mathrm{k}=-1$. That is, 481 is the divisor of $\mathrm{A}$ if and only if 481 divides $\mathrm{B}=\sum_{i=1}^{n} 10^{i} a_{i}-48 . a_{0} ; \mathrm{B}=625-48(3)=481$, thus, $\mathrm{A}=6253$ is divisible by 481 .

Example 4.2.

Consider $m=2600$ the divisor, clearly, not co-prime to 10 and $A=27300$. Then, the prime factorization: $2600=2^{3} \cdot 5^{2} \cdot 13 . A \equiv 0(\bmod 2600) \quad$ if $300 \equiv 0(\bmod 8), 300 \equiv 0(\bmod 25)$ and $2730-9 . a_{0} \equiv$ $0(\bmod 13)$. But, 2600 does not divide 27300 , because 8 does not divide 300 . Though, as $2730-9 * 0=2730,273-$ $9 * 0=273$, and $27-9 * 3=27-27=0$ where 13 divides 0 , so , 13 divides 27300 , and as the last two digits are 0 and 25 divides 0,25 divides 27300

\section{Conclusion}

In this paper, firstly, we showed that the method to dividing number by a positive number a positive number $m$ co-prime to 10 . And eventually, we generalized the test to divisibility by any positive number $m$ not relatively prime to 10 , considering its standard factorization in which at least $2^{\alpha}$ or $5^{\beta}$ is a factor to $m$ and evidently any factor $h \neq 2^{\alpha}, 5^{\beta}$ and $2^{\alpha} .5^{\beta}$, is co-prime to m. Now, let $m=2^{\alpha} \cdot 5^{\beta} . h$ be a factorization of $\mathbf{n}$ and $A=a_{n} a_{n-1} a_{n-2} \ldots a_{2} a_{1} a_{0}$ be a positive integer in decimal system, where $0 \leq a_{0}, a_{1}, a_{2}, \ldots \ldots, a_{n} \leq 9$ are integers. Then $\mathrm{m}$ divides $\mathrm{A}$ if and only if $\mathrm{m}$ divides $\mathrm{B}$, where

i. If $\alpha, \beta=0, \mathbf{n}$ is co-prime to 10 , then $\mathrm{B}=\sum_{i=1}^{n} 10^{i} a_{i}+\left(\frac{1+m k}{10}\right) \cdot a_{0}, k \in Z^{-}$

ii. If $\alpha$ or $\beta \neq 0$, $\mathbf{n}$ must divide the number formed by the last $\alpha$ or $\beta$ digits $A$ and as $h$ is co-prime to 10 , we use the case of (i) to $h$.

That is, the number $\mathbf{A}$ is divisible by $\mathbf{m}$, if and only if it is divisible by $2^{\alpha}$ or $5^{\beta}$, and $h$.

\section{References}

Burton, D. M. (2006). Elementary Number Theory (second ed.). Tata: MAcgraw-Hill Education.

Koshy, T. (2007). Elementary Number Theory with Application. New York: Academic Press.

Rosen, K. I. (1982). A Classical Introduction to Modern Number Theory (2nd ed.). New York: Springer-Verlag. 
Shoup, V. (2005). A Computational introduction to number theory. Cambridge university press. Stein, W. (2004). Elementary Number Theory. Harvard: Harvard University Press.

Weisstein, E. (2015, may 3). congruence. MathWorld-A wolfram Web Resource, pp. 45-56. 